\title{
Ultrasound Biomicroscopy in Small Animal Research: Applications in Molecular and Preclinical Imaging
}

\author{
A. Greco, ${ }^{1,2,3}$ M. Mancini, ${ }^{3,4}$ S. Gargiulo, ${ }^{1,2}$ M. Gramanzini, ${ }^{2,3}$ P. P. Claudio,, 6 \\ A. Brunetti, $1,2,3$ and M. Salvatore ${ }^{1,4}$ \\ ${ }^{1}$ Department of Biomorphological and Functional Sciences, University of Naples “Federico II", 80131 Naples, Italy \\ ${ }^{2}$ CEINGE-Biotecnologie Avanzate, s.c.ar.l., 80145 Naples, Italy \\ ${ }^{3}$ Institute of Biostructure and Bioimaging, Italian National Research Council (CNR), 80131 Naples, Italy \\ ${ }^{4}$ SDN Foundation Instituto di Ricovero e Cura a Carattere Scientifico (M.M.), 80131 Naples, Italy \\ ${ }^{5}$ Department of Biochemistry and Microbiology, Joan C. Edwards School of Medicine, Marshall University, Huntington, \\ WV 25755, USA \\ ${ }^{6}$ Department of Surgery, Joan C. Edwards School of Medicine, Marshall University, Huntington, \\ WV 25755, USA
}

Correspondence should be addressed to A. Greco, adegreco@unina.it

Received 1 August 2011; Accepted 12 August 2011

Academic Editor: Monica Fedele

Copyright ( 2012 A. Greco et al. This is an open access article distributed under the Creative Commons Attribution License, which permits unrestricted use, distribution, and reproduction in any medium, provided the original work is properly cited.

\begin{abstract}
Ultrasound biomicroscopy (UBM) is a noninvasive multimodality technique that allows high-resolution imaging in mice. It is affordable, widely available, and portable. When it is coupled to Doppler ultrasound with color and power Doppler, it can be used to quantify blood flow and to image microcirculation as well as the response of tumor blood supply to cancer therapy. Target contrast ultrasound combines ultrasound with novel molecular targeted contrast agent to assess biological processes at molecular level. UBM is useful to investigate the growth and differentiation of tumors as well as to detect early molecular expression of cancer-related biomarkers in vivo and to monitor the effects of cancer therapies. It can be also used to visualize the embryological development of mice in uterus or to examine their cardiovascular development. The availability of real-time imaging of mice anatomy allows performing aspiration procedures under ultrasound guidance as well as the microinjection of cells, viruses, or other agents into precise locations. This paper will describe some basic principles of high-resolution imaging equipment, and the most important applications in molecular and preclinical imaging in small animal research.
\end{abstract}

\section{Introduction}

Mice are widely used as models for studying many human diseases. The main advantage of research conducted in small animals is owed to their short life cycle and the possibility of genetic manipulation. However, most of the observations in small animals have been based in the past on surgery and histological postmortem analysis. Few years ago, research applications of noninvasive imaging methods such as optical imaging, computed tomography, magnetic resonance, micro-PET-SPECT, and ultrasound were limited to larger animals such as dogs and nonhuman primates. In the recent years, a new ultrasound technology, called ultrasound biomicroscopy (UBM) was optimized to evaluate animal models of human disease. UBM is a noninvasive realtime technique that allows accurate and reliable images of the heart and other organs in mice [1-3]. Additionally, this technique is useful to image the fetal mouse [4-6] and to obtain high-resolution images of mice tumors $[7,8]$. The consistent ultrasound image obtained with the UBM can be used to visualize and guide injection into target organs $[9,10]$, including mouse embryo, to aid in targeted delivery of drugs and viral particles [7, 11]. UBM allows longitudinal data acquisition at low cost and noninvasively to investigate the growth and differentiation of tumors as well as to monitor the effects of cancer therapies; therefore, it reduces the number of animals needed to perform experiments [12].

The basic modalities include B-mode, M-mode, Doppler mode, $3 \mathrm{D}$ reconstruction, power mode, and an ECG-based Kilo-Herz visualization technology. Doppler ultrasound with color and power Doppler can be used to quantify blood flow 
and to image microcirculation and the response of a tumor blood supply to cancer therapy [13]. Recently, the introduction of ultrasound contrast agents (lipid shell gas-filled 14 micron sized microbubbles) enhances UBM applications for detection and characterization of focal lesions. Recently, the introduction of ultrasound contrast microbubbles that is targeted to molecular markers expressed on the vasculature is able to image molecular events of disease and could be used for various applications including quantitative analysis of molecular biomarkers, perfusion studies, microvasculature targeting, and gene and drug delivery $[7,11]$.

Current applications of UBM are the following:

(i) studies on mouse development from early embryonic period to adulthood,

(ii) in vivo morphological and functional phenotyping of wild-type, transgenic and mutant mice and other mouse disease models,

(iii) tumor growth monitoring,

(iv) evaluation of effects of therapeutic interventions,

(v) imaging-guided intervention on mice,

(vi) microinjection for introducing genes or cells into the developing mouse embryos and follow-up of the effects,

(vii) ultrasound-guided catheterization (veins or bladder).

This paper describe basic principles of high-resolution imaging equipment and some applications in molecular and preclinical imaging in small animal research.

\section{UBM Methodology}

Human ultrasound scanners are limited to $2-20 \mathrm{MHz}$ frequencies, since deep penetration and a axial spatial resolutions of $0.2-1 \mathrm{~mm}$ is required. UBM uses frequencies of 40-100 MHz. Higher frequencies are used to image cellular structures: the scanning acoustic microscopy (SAM) uses frequencies of $100 \mathrm{MHz}-1 \mathrm{GHz}$ for the evaluation of thickly sliced biological tissue or cells [14] and explore the acoustic properties of single cells with submicrometric resolution.

The choice of ultrasound frequency and the type of transducer represents a balance between image resolution and penetration depth.

The high-frequency ultrasound $(20-100 \mathrm{MHz}$ range) is used in mice imaging with mechanical sector scanhead with fixed focus or with electric probes with multiple focus. These transducers allow high-resolution imaging and require a mechanical support to perform the necessary micromovements for mice examinations (Figure 1).

At these frequencies, sound waves are transmitted through soft tissue relatively to the acoustic impedance of each tissues. The acoustic impedance of a particular tissue is the product of sound transmission velocity and tissue density. The transmission velocity in most soft tissue is nearly uniform at $1540 \mathrm{~m} / \mathrm{s}$; therefore, the acoustic impedance of most soft tissue is primarily a function of tissue density. In the frequency ranging from 20 to $100 \mathrm{MHz}$, only few studies of attenuation, backscatter, and speed of sound have been performed. The frequency dependence of attenuation is strongly dependence on the tissue type. The mechanism of the differences between various transducers is not well understood, but it is known that the concentration of collagen and other structural protein are of primary importance. In the case of a homogeneous soft tissue, an attenuation coefficient of $6.5 \mathrm{~dB} / \mathrm{mm}$ can be expected at $60 \mathrm{MHz}$ probe frequency [15]; therefore, a penetration of $6 \mathrm{~mm}$ would be expected for a system dynamic range of $80 \mathrm{~dB}$.

Backscatter from blood at lower frequencies used in human diagnostic applications is predominantly dependent on shear rate due to the formation of red cells aggregates called "Roleaux." At frequencies greater than $40 \mathrm{MHz}$, the backscatter appears less dependent on shear rate, because the red blood cells begin to act as mirroring reflectors. At higher frequencies, the lumen of the vessel becomes isoechoic with the surrounding tissue, impeding accurate boundary discrimination. This natural enhancement of the blood signal is a benefit for Doppler analysis but at the expense of B-mode imaging quality.

Resolution is the ability to accurately distinguish two closely situated structures and becomes a crucial factor when imaging small targets like mouse organs (the diameter of an internal carotid artery is $0.2 \mathrm{~mm}$, and the left ventricular posterior wall is approximately $1 \mathrm{~mm}$ thick [16]. Axial and lateral resolution improves with increasing frequencies. The axial resolution is dependent of the pulse spatial length and, therefore, for fixed cycles number is due to the wavelength.

The lateral resolution is the product of the transducer diameter, focal length, f-number, and wavelength. Therefore, the lateral resolution depends from transducers geometry and wavelength. The f-number equals the depth of returning echo (focal length) divided by the aperture of the beam (transducer diameter). It will be best (smallest) if there is a large aperture of the transducers and short wavelength (higher frequency).

Utilizing frequency of $40-60 \mathrm{MHz}$ and a narrow beam width, a spatial resolution of 30-50 microns can be obtained. At these frequencies, the penetration is reduced to $1-2 \mathrm{~cm}$. Hence, increasing axial and lateral resolution by increasing the frequency limits the depth penetration; however, this does not affect mice imaging, because most of the target organs are in the penetration depth range of the high frequency used.

Temporal resolution is the ability to distinguish two events in time and is an important factor when imaging mice hearts that have a heart rate of 400-600 beats/min. At heart rate of 400 beats/min, a frame rate of $30 \mathrm{~Hz}$ would result in only about 4 images for each cardiac cycle. Each image frame acquires $25 \%$ of the cardiac cycle, making the determination of the systolic and diastolic phase inaccurate. High-frequency ultrasound has low imaging frame rates $(5-10 \mathrm{~Hz})$ with relatively poor temporal resolution for moving structures. It is not important when imaging static or slow moving organs such as liver or xenografts, but it is indispensable for the evaluation of the heart. The Kilo-Herz visualization technology use B-mode frame synchronized with ECG trace that produce a postprocessing imaging equal to a real-time $1,000 \mathrm{~Hz}$ frame rate. 


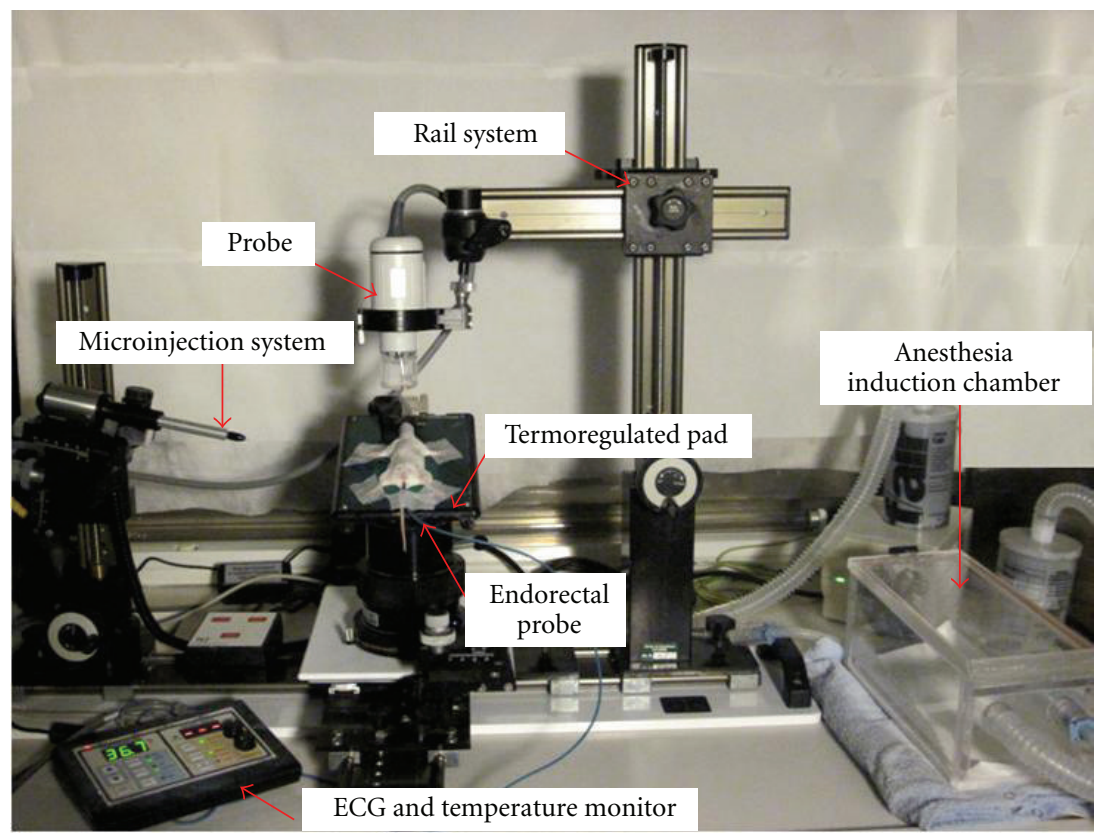

FIGURE 1: Ultrasound biomicroscopy integrated mechanical support with rail system. The microinjection system for visualization and guidance of injection and extraction procedures in real time. Ultrasound probe is mounted on a system securing the probe in a stationary position when the ultrasound scan is in the desired image plane. After anesthesia, the mouse is positioned on a termoregulated pad to monitor ECG and temperature (with endorectal probe) and ensure mouse comfort during imaging.

The UBM Doppler system using a typical duplex Doppler configuration operates at $20-55 \mathrm{MHz}$ with a pulse repetition frequency of $1-20 \mathrm{KHz}$. The transducer is held stationary to obtain Doppler flow velocity spectra in real time for a sample volume located on the B-mode image. The default setting of Doppler system are pulse repetition frequency (PRF) from 1.0 to $120.0 \mathrm{kHz}$ (transmit setting), the wall filter from 0 to $1000 \mathrm{~Hz}$, and the Doppler gain from 1.0 to $10 \mathrm{~dB}$ (receive settings).

The Doppler sample volume size changes with the number of cycle per pulse and insonation frequency from 2.18 to $4.36 \mu \mathrm{L}$ for $20 \mathrm{MHz}$ to 0.46 to $0.93 \mu \mathrm{L}$ for $40 \mathrm{MHz}$ and 8-16 numbers of cycles per pulse. The high-frequency Doppler system is able to measure velocity from $37 \mathrm{~cm} / \mathrm{sec}$ ( $\max$ analyzed velocity at $0^{\circ}$ ) to a minimum velocity of $1 \mathrm{~mm} / \mathrm{sec}$. The system is able to detect low blood velocities in small vessels, and it can calculate blood flow with dedicated software.

3D UBM imaging allows viewing of the tissue of interest as a whole organ in different orientations. This application in experimental medicine can be useful in the detection of xenograft tumors in mice and in longitudinal growth. A set of consecutive 2D image planes of the tumor are acquired then reconstructed into $3 \mathrm{D}$ views for tumor estimation analysis $[17,18]$.

An automated method acquires the images, using dedicated 2D transducers. The probe is mounted on a rail system equipped with a 3D motor stage.

Based on operator-defined parameters, the 3D motor stage travels a set distance across the target object in a series of tiny steps. At each step, the probe takes a twodimensional "slice." Each two-dimensional B-mode image slice is assembled with the other slices of acquired data and rendered by the software into a three-dimensional data set. The digitally stored volume data can be presented in a three-dimensional view of the acquired data (cube view), a three single, slidable image slice views (cross view), "transverse view," straight-on perspective of the $x-y$ plane image slice, "sagittal view" and "coronal view" (Figure 2). $3 \mathrm{D}$ volumes can be created in $3 \mathrm{D}$-mode or power $3 \mathrm{D}$ mode using parallel or rotational segmentation. For either method of segmentation, the system can perform a manual or semiautomated segmentation of the volume. When the manual segmentation procedure is followed, the operator draws each contour of the volume. After the semiautomated segmentation procedure is followed, the system draws two or more contours automatically.

Vascularization of tissues within the region of interest can also be assessed using 3D power-Doppler ultrasound and a percent vascularity value (PV) is provided after the volume has been created. The PV provides the percentage of the volume that contains flow detected from power Doppler mode.

\section{Contrast Agent}

The most used US contrast agents (both in preclinical and clinical research) consist of small, stabilized gasencapsulated microparticles $(<10 \mu \mathrm{m})$ defined microbubble contrast agents that we injected into the bloodstream behave similarly to red blood cells in the microcirculation providing a strongly reflective blood/gas interface. This allows the detection of purely intravascular molecular targets, whereas intracellular molecular events cannot be 


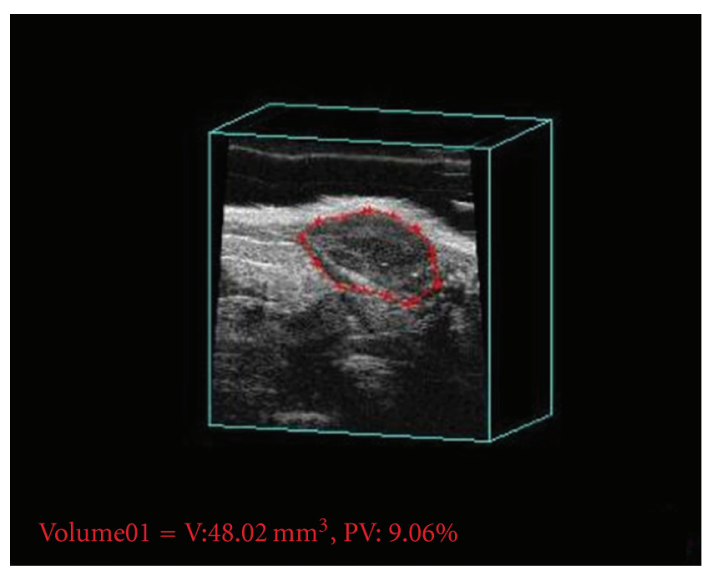

(a)

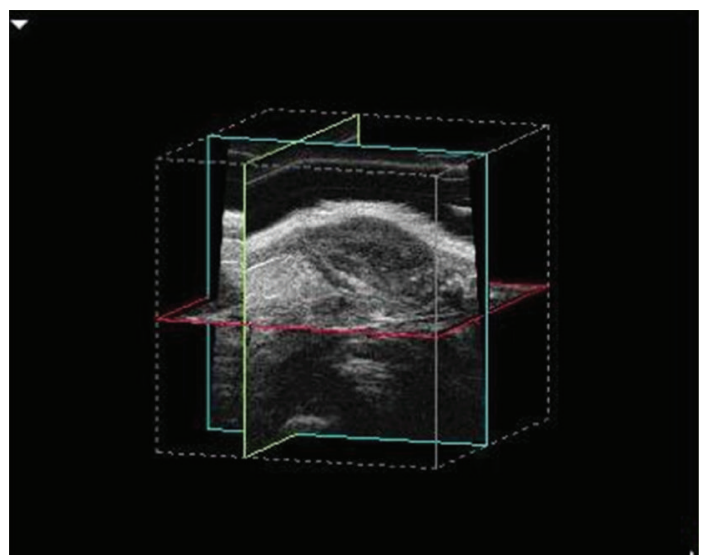

(c)

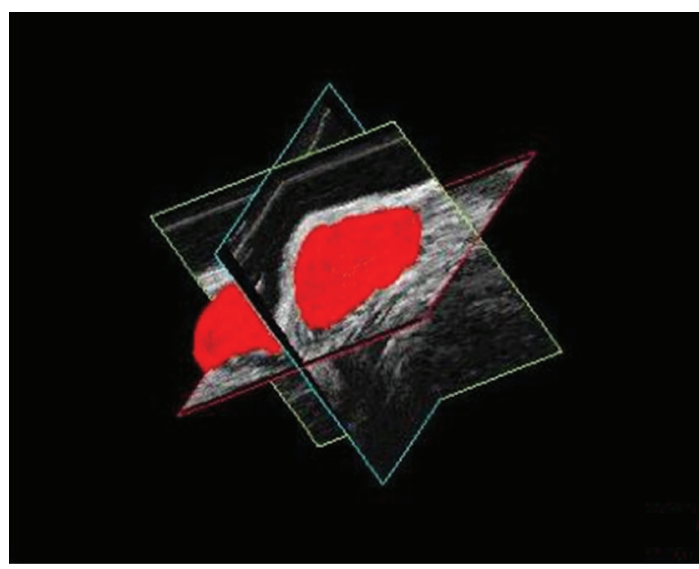

(b)

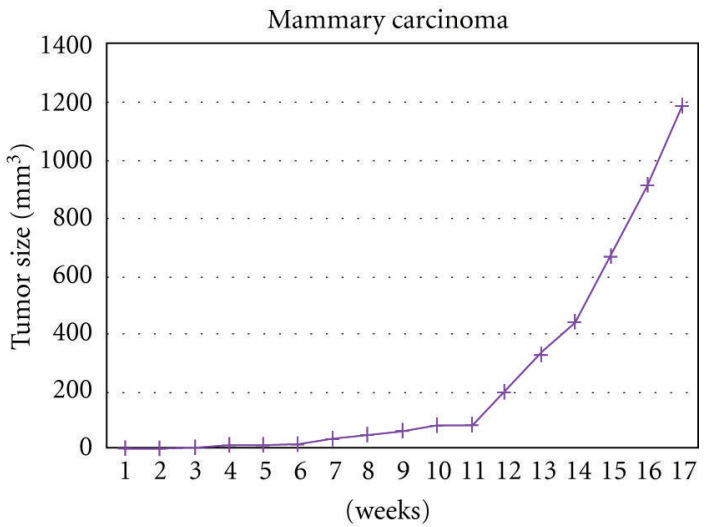

(d)

FIGURE 2: Various view of three-dimensional reconstructions of a subcutaneous tumor from two bidimensional successive parallel crosssection images. (a) Cube view. On the bottom, the segmentational volumetric analysis with a volume estimation of $48.02 \mathrm{~mm}^{3}$. (b) Surface view. (c) Cross view. (d) Quantitative analysis of tumor growth in a mice model of mammary tumor. The tumor growth curve over time is showed in the diagram.

imaged. The microbubbles signal is dependent on a number of factors such as the filling gas property, for example, compressibility and solubility, the surrounding medium properties as viscosity and density, the frequency and power of ultrasound applied, the bubble size, and shell properties as elastic modules, thickness, and damping effects. The behavior of microbubbles depends on the amplitude of ultrasound to which they are exposed. A very low acoustic power (mechanical index $<0.05-0.1$ ) produces ultrasound scattering equal to the transmitted frequency. A slightly higher mechanical index of $0.1-0.3$ produces a backscatter of a variety of frequencies (harmonics, subharmonics, and ultraharmonics). Higher acoustic pressures (MI >0.3-0.6) destroy the microbubbles (Figure 3). This occurrence produces a high-intensity broadband signal, changes in the microenvironment, or even shell ballistic events that are important features for both perfusion imaging and therapeutic applications as local delivery of drug or genes.

It is possible to target microbubbles to specific region of disease. Actually, there are two mechanisms for targeting the contrast agent: a passive and an active mechanism of targeting.

The passive targeting mechanism is based on an unspecific electrostatic or chemical interaction between the microbubble shell and the receptors of the endothelial wall expressed by diseased tissues.

The active targeting mechanism is based on the specific binding between shell microbubbles' ligands and surface disease antigens. The "active targeting" uses the attachment of specific antibodies, such as anti-VEGF for example, to the surface of microbubbles with streptavidine-biotin interaction (Figure 4). This leads to the accumulation of targeted contrast agent to specific sites due to the use of adhesion ligands including antibodies, peptides, and polysaccharides. Since the microbubbles remain within the vasculature, because of their size in the micron range, specific marker molecules have to be located in the intravascular space and on the endothelium to be targeted during pathological events. Specific ultrasound imaging protocols are used to detect retention of targeted microbubbles (Figure 5). After the bolus injection of the targeted ultrasound contrast agent, 


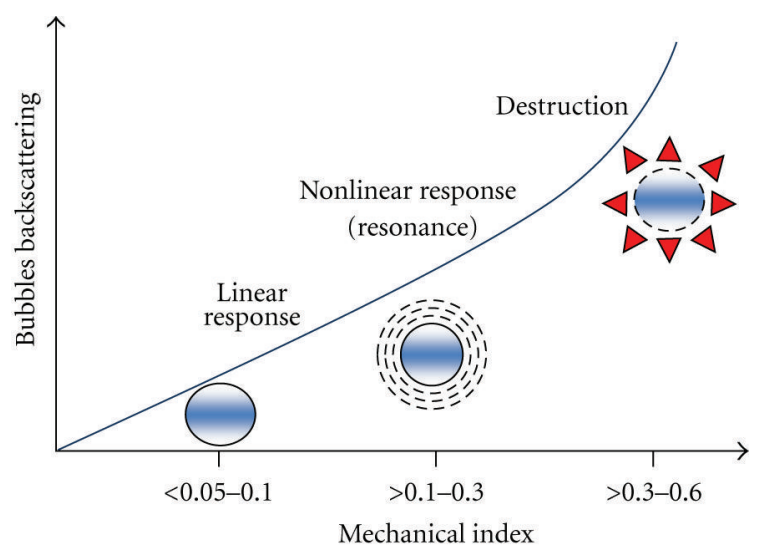

Figure 3: Behavior of microbubbles depends on the amplitude of ultrasound to which they are exposed. At very low acoustic power (mechanical index $<0.05-0.1$ ), microbubble oscillates in relatively symmetrical backscattering at the same frequency of incident ultrasound. At a slightly higher mechanical index of $0.1-$ 0.3 , the microbubble becomes somewhat oscillates in a nonlinear manner (nonlinear response), backscattering a variety of frequencies (harmonic). Higher acoustic pressures (MI > 0.3-0.6) destroy the microbubbles with high-intensity backscatter response.

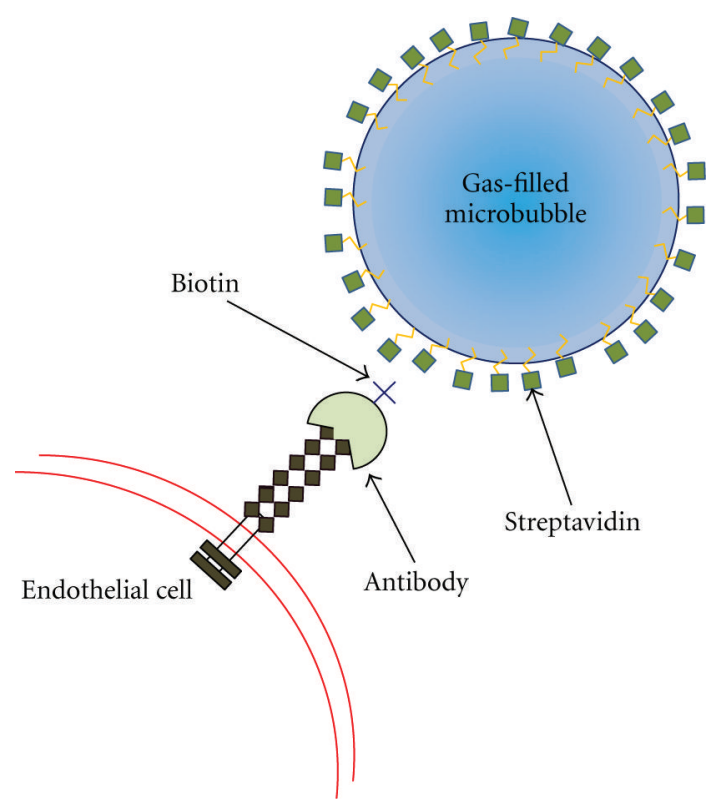

FIgURE 4: Schematic representation of active targeting of biotinstreptavidine bridges. Streptavidine is used for attachment of biotinylated ligands onto the shell of ultrasound contrast microbubbles. Molecularly targeted microbubbles selective bind to sites of molecular expression on the endothelium.

it is necessary to wait for 4 to 15 minutes before starting the contrast-enhanced imaging. This elapsed time allows retention and clearance of most circulating microbubbles from the blood pool. Sets of 300 frames (temporal resolution of $30 \mathrm{~Hz}$ in a period of 10 seconds) are then captured to obtain a signal from the tumor tissue background and contrast agent accumulated in the target site both retained and freely circulating microbubbles. Therefore, using destruction-subtraction algorithm the residual circulating contrast signal is measured. After destruction of microbubbles by 2 to 3 seconds of "break" frames performed with high mechanical index, a new set of 300 frames is captured to derive the signal only from tumor tissue and freely circulating microbubbles. The images acquired before breaking pulses and after breaking pulses are averaged and digitally subtracted to derive the signal representing only retained microbubbles. Late imaging modality of single intravenous injection of contrast media optimizes the signal coming from retained microbubbles with respect to the one coming from freely circulating microbubbles, since these latter are gradually removed from the blood pool. The signal from freely circulating microbubbles is very low, accounting for $15 \%$ of the contrast signal intensity on initial frames with the targeted agents. The entire imaging procedure typically lasts 30 minutes, then data analysis is performed offline, consisting mainly of digital subtraction and frame alignment, region-of-interest quantification and color map processing. Many researchers have used microbubbles in small animals for the detection of molecular markers of inflammation, angiogenesis, and atherosclerosis [19-22].

Ultrasound contrast agents are viable candidates for gene delivery/therapy. Ultrasound energy determines an increase of cell membrane permeability (a process known as sonoporation), which is being increasingly exploited for its role in drug-delivery applications to transfer therapeutic agents including genetic material, proteins, plasmid DNA, and chemotherapeutic agents, directly to the pathological tissue and organs. The use of US is based on the fact that the contrast agent can be identified at the target site and it can readily be insonated causing ultrasound-induced rupture of the drug-loaded microbubbles hence achieving targeted drug release. Most targeted ultrasound contrast agents are microbubbles, but other vehicles can be used including acoustically active liposomes and perfluorocarbon emulsions.

Echogenic liposomes can readily be conjugated to antibodies or other adhesion ligands, and thus are readily configured as targeted agents. Liposomes, are less than $1 \mathrm{~mm}$ in diameter and due to the small diameter, these agents are not entrapped in the microvasculature of the lung and have a long circulating time. Additionally, the liquid-like composition of liposomes makes them more resistant to pressure and mechanical stress than microbubbles. Another advantage of liposomes have been used to entrap gas and drugs for ultrasound controlled drug release and ultrasoundenhanced drug delivery [23]. Echogenic liposomes have been produced by different preparation methods, including lyophilization, pressurization, and biotin-avidin binding [24]. Additional benefits of liposomes are that lipids are small molecular structures and the lipid complexes can be made smaller by filtering or sonication techniques. Proteins and delivery agents made of proteins tend to break if they are manipulated to make them smaller. The difference lies in the rigidity of the protein, which has covalent bonds, versus the lipid, which is composed of small molecules held together by hydrophobic interactions [25]. 


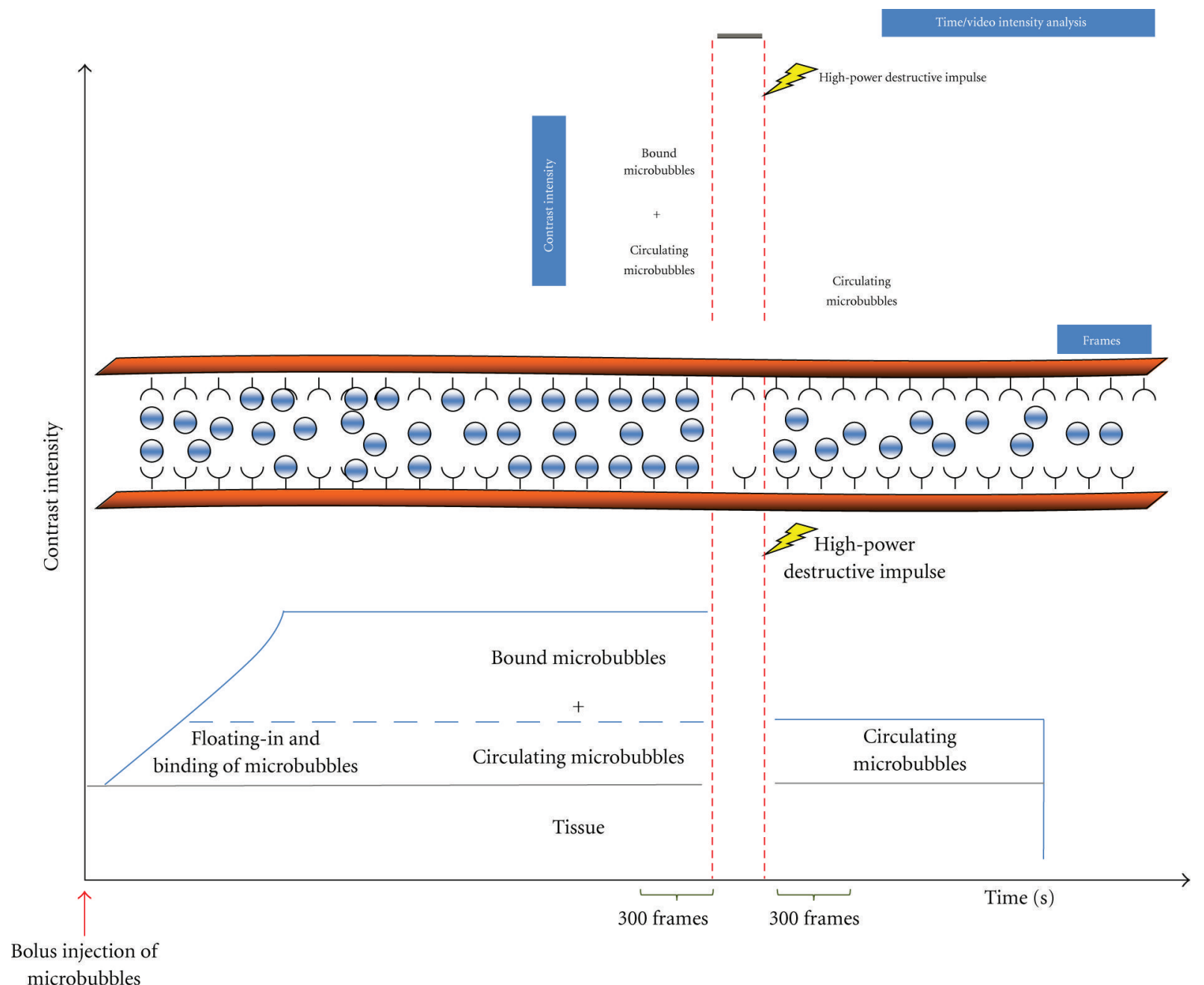

microbubbles

$\bigcirc$ Microbubbles

$\Psi$ Endothelial cells

High-power destructive pulse

FIGURE 5: Targeted US of endothelial antigens in vessels of a tumor tissue. On the top of the figure time/video intensity analysis before and after high-power destructive pulse and bottom a diagram representation of destructive methodology. Endothelial cells of vessels (orange) of tumor tissues expresses specific antigens. After intravenous administration targeted microbubbles float in vessels and remaining exclusively in the vascular compartment. Many of them bind to antigens of endothelial cells, whereas others remains in the vessel lumen freely circulating. After high-power destructive pulse, all microbubbles are destroyed (bound + circulating), following circulating microbubbles that arrive from outside of scan plane, which remain freely circulating for several seconds. Contrast intensity is the sum of the intensity from tissue, intensity from microbubbles not bound to receptors (circulating microbubbles), and intensity from microbubbles bound to receptors on endothelial cells. After digital subtraction of the video intensity calculated on 300 predestruction frames from video intensity calculated on 300 postdestruction frames, resulting video intensity is due only to bound microbubbles.

Perfluorocarbon emulsion nanoparticles can be used as an ultrasound contrast agent. Due to their size (approximately $250 \mathrm{~nm}$ ) in diameter and composition, these agents have the same qualities of gas encapsulated microbubble contrast agent and the liposomes [23, 26]. These nanoparticles avoid lung entrapment. They consist of a lipid encapsulated perfluorocarbon, which is a liquid at room temperature. The liquid composition makes it resistant to pressure and mechanical stress. However, due to their small size, their echogenicity is weak until they are deposited in a layer. This can be an advantage, as the low free-floating echogenicity results in a decreased background noise level. Submicron particles filled with liquid perfluorocarbon are currently being studied as a potential ultrasound targeted contrast agent.

Nanoparticles with directly conjugated tissue factor antibody demonstrated acoustic enhancement similar to that demonstrated with the avidin-biotin targeting approach with the advantage of one step targeting method [27].

Recently, acoustically activated submicron droplets of liquid perfluorocarbon have investigated as a "new class" of ultrasound contrast agent. In the liquid state, intravascular droplets can extrasavasated within tumors, resulting a candidate for an extravascular ultrasound contrast agent. 
TABLE 1: Specific procedures related to UBM.

\begin{tabular}{llc}
\hline Procedures & Applications & References \\
\hline $\begin{array}{l}\text { Vein injection (jugular vein, femoral vein, } \\
\text { and tail vein) }\end{array}$ & Contrast agent administration in single bolus & {$[34]$} \\
\hline Blood vessels cannulation & Genes and drugs delivery & {$[31]$} \\
\hline & Contrast agent administration in repeated bolus & {$[31,32,35,36]$} \\
\hline \multirow{3}{*}{ Ultrasound-guided microinjections } & Viral vectors and gene delivery & {$[37]$} \\
& Drugs delivery & {$[38]$} \\
& Guided in utero microinjection & {$[33,39,40]$} \\
\hline Biopsy & Stem cells therapy & {$[9]$} \\
\hline
\end{tabular}

Activation was accomplished by using burst of ultrasound to vaporize the droplets [28].

Finally, Bekeredjan et al. tested gold-bound microtubules to provide a backscattering that allowed microtubules to be potentially useful as an ultrasound contrast agent. Goldbound microtubules provide a persistent contrast effect, suggesting their use as an ultrasonic contrast agent with the feasibility of antibody conjugation [29].

\section{Animals Preparation to UBM Studies}

Mice are anesthetized using 1.5\%-2\% isoflurane vaporized in oxygen with the aid of a precision vaporizer (we used the vaporizer manufactured by Vetequip Inc., Pleasanton, Calif, USA) to deliver the appropriate amount of anesthetic, with constant monitoring of their body temperature (with a rectal probe) and heart rate. Body temperature is maintained at $35^{\circ} \mathrm{C}-38^{\circ} \mathrm{C}$ using an infrared lamp and a heating pad. Hair is removed from the area of interest with a chemical hair remover to obtain a direct contact of the ultrasound gel to the skin of the animal and to minimize US attenuation. To provide a coupling medium for the transducer a prewarmed low-density ultrasound gel is used (we utilize the Aquasonic 100 ultrasound transmission gel, Parker, USA). Using all these cautions is possible to perform a safe UBM study on mice without particular regards to the anesthesia duration. The position of the two-dimensional image plane is obtained with the ultrasound probe fixed to a rail system that allows repeatable and precise examinations (Vevo Integrated rail System II).

\section{Specific UBM Procedures}

Some surgery procedures on mice can be necessary during studies: tail vein injection, cannulations, and microinjections (Table 1).

Tail vein injection is used to perform contrast UBM imaging for perfusion studies, for the administration of drugs or genetic materials contained in microbubbles. Before the injection, a vasodilatation is induced to better localize the lateral tail vein. It is important to avoid injection exceeding $200 \mu \mathrm{L}$ to avoid hypervolaemia and lung edema.

Cannulation has several advantages compared to tail vein injection: it allows making injections in the jugular vein, and it is less traumatic for wall vessels and allows controlled and repeated injections during the same imaging study without changing the scanning plane.

Cannulation of the jugular vein [30] is an established method for studying drug pharmacokinetics and effects. After anesthesia, the skin between the ears and the righthand side of the neck of the mouse needs to be shaven and the skin disinfected. A longitudinal incision of about $15 \mathrm{~mm}$ is made in the neck of the animal. After careful removal of the connective tissue surrounding the jugular vein, the cannula, filled with heparin solution $(750$ units $/ \mathrm{mL}$ in water), is inserted into the vein in the caudal direction. The cannula is fixed to the jugular vein by ligation. Finally, the incision in the neck of the animal is closed using 5/0 Ethilon silk suture and a metal spring is firmly attached to the stitches (Figure 6(a)).

Cannulation of the lateral tail veins $[31,32]$ can also be performed in the mouse to inject contrast ultrasound agent. Animals are immobilized by taping the tail to an $18 \mathrm{~cm} \times$ $7 \mathrm{~cm}$ plexiglass rectangle and by fastening a restraint tube (125 mL half-Nalgene bottle) using rubber bands. The tail is then washed and immersed in warm water $\left(42^{\circ} \mathrm{C}\right)$ for approximately 45 seconds to dilate the tail veins. After this, the tail is cleaned and wiped with ethanol. A 27-gauge needle connected by PE 10 tubing to a $1 \mathrm{~mL}$ saline-filled syringe is inserted into the right lateral vein approximately $2 \mathrm{~cm}$ from the body and its intravascular position confirmed by drawing blood (Figure 6(b)). The needle is then removed, and a saline-heparin- (SH; $150 \mathrm{IU} / \mathrm{mL}$ ) filled catheter is introduced through the access site. Proper position of the catheter in the vein is verified by the observation of blood backflow. The catheter is then secured to the tail using cyanoacrylate glue (we use Histoacryl, B Braun, Am Aesculap-Platz, Germany) at the point of insertion.

UBM-guided injections, such as targeted injections of retroviruses, cells, or genetic materials in mouse embryos, can be done manually [10] or by mechanical guide [33] and can be performed transcutaneously or with surgical exposure of the target organ. Injections can be performed with $50 \mu \mathrm{L}$ Hamilton syringe and a 30 gauge needle [10] or with microinjector units and capillary glass needle that can inject very small volume (about $10 \mathrm{~nL}$ ).

\section{In Utero Microinjections}

In utero ultrasound-guided microinjection of mouse embryos has proven a valuable tool for exploring the 


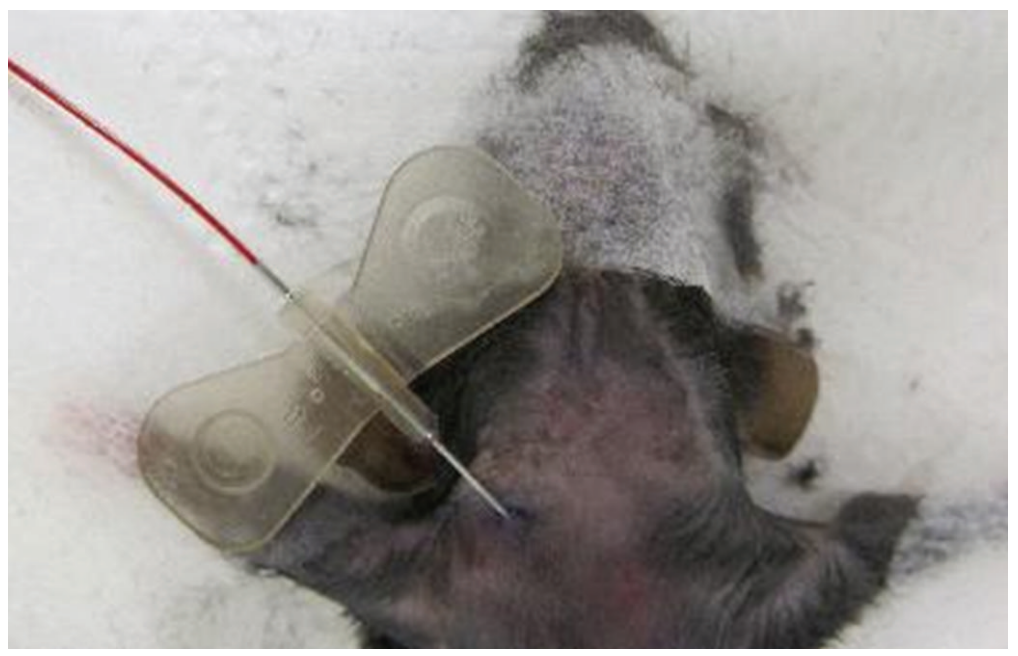

(a)

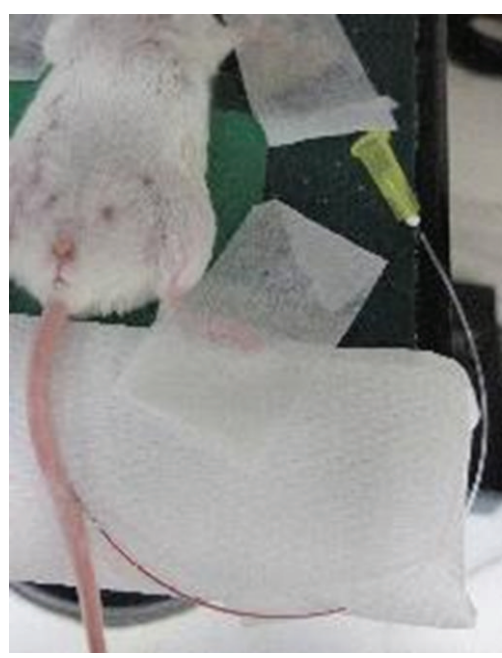

(b)

FIgURE 6: Cannulation of jugular and tail veins. The anesthetized mouse is immobilized by taping its harms on a termoregulated pad. A side of the neck or the tail of the mouse are disinfected. A 27-gauge needle connected by polyethylene tubing (PE10) to a $1 \mathrm{~mL}$ saline-filled cannula is inserted into the jugular vein or in the lateral tail vein approximately $2 \mathrm{~cm}$ from the body. The cannula, filled with heparin solution (750 units/mL in water), is inserted into the vein in caudal direction and fixed to the vein with cyanoacrylate glue (Histoacryl, B Braun, Am Aesculap-Platz, Germany). The proof of the correct position of the needle is given by blood visualization in the cannula. (a) Cannulation of jugular vein. (b) Cannulation of lateral tail vein.

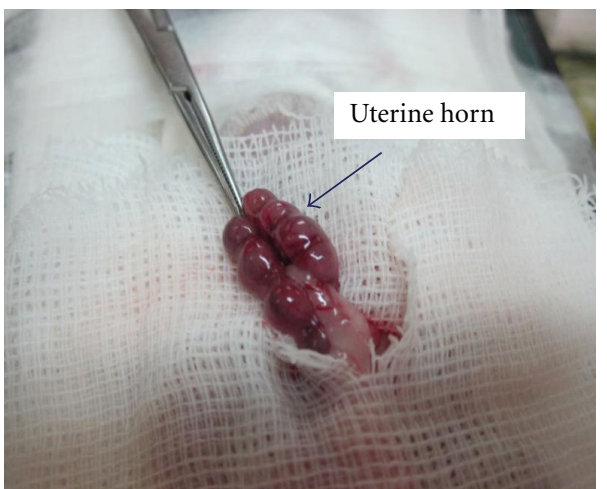

(a)

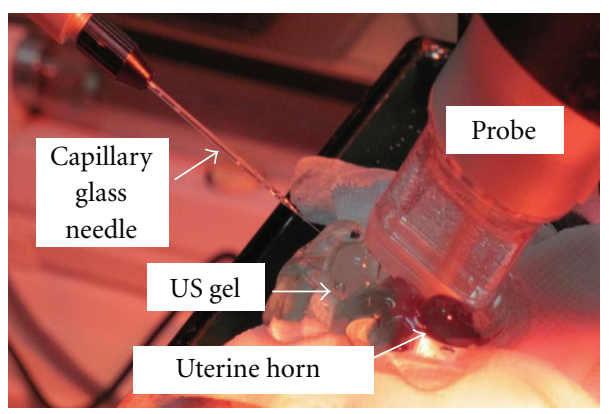

(b)

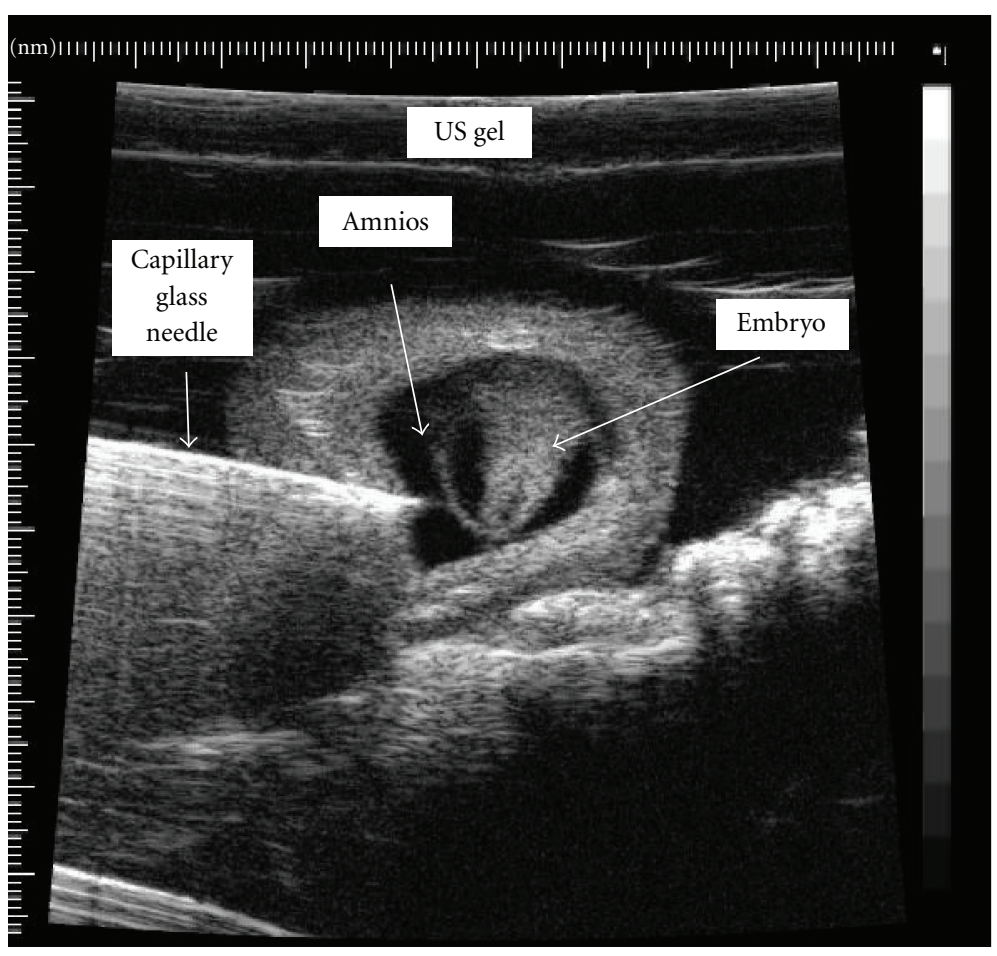

(c)

FIGURE 7: In utero microinjection: laparoscopy along the linea alba. The uterine horns are gently exteriorized to record implantation sites and simply positioned on sterile gauze and covered with sterile acoustic gel (a). A $40 \mathrm{Mhz}$ probe is used to image in real time the embryos, and the microinjection is performed by an automatic microinjector equipped with a capillary glass needle. The needle is advanced with the use a micromanipulator under echo guidance until the needle tip is in the desired location (b). UBM images of a capillary glass needle advanced under echo guidance until the needle tip is in the amnions (c). 
developmental consequences of altering gene expression using viral vectors and for injecting cells to study cell lineage or migration and to validate gene therapies. Pregnant females need to be monitored during anesthesia. Physiological parameters such as heart rate, respiratory frequency, and temperature are useful to assess anesthetic depth during the procedure and the maternal-fetal wellbeing. Microinjection procedure contemplate a laparoscopy along the linea alba after skin disinfection with $70 \%$ ethanol. The mouse is mounted on the stage of a rail system, which permits the operator to readily adjust the position of the mouse while maintaining the microinjection pipette within the transducer-imaging plane. The uterine horns are gently exteriorized to record implantation sites and simply positioned on sterile gauze and covered with sterile acoustic gel (Figure $7(\mathrm{a})$ ).

A $40 \mathrm{Mhz}$ probe is used to image in real time the embryos, and microinjection is performed with an automatic microinjector equipped with a capillary glass needle. The needle is advanced with the use a micromanipulator under echo guidance until the needle tip is in the desired location (Figure $7(\mathrm{~b})$ ). In this way, the procedure is less traumatic for the embryos, and a very small amounts of material can be injected, significantly reducing the risk of embryonic death. After microinjecting all sites, the gel is removed with a squeeze bottle containing sterile PBS, and embryos are placed back into the body cavity. The maternal abdomen is then closed using a 8-0 silk suture using a continuous stitch up, anesthesia is discontinued, and the mouse is placed in a heated recovery chamber. UBM is performed in the following two days to test the fetuses' health status.

Commonly, microinjection is performed at embryonic day 9.5 (E9.5) or greater, but recently, with advances in technology, it has been reported the possibility to accurately target specific regions of the embryos under high-resolution ultrasound guidance at E6.5 to E7.5 of gestation [33].

\section{Applications}

7.1. Oncology. High-Frequency ultrasound represents a significant advancement in the phenotypic assessment of mouse cancer models. Tumor size can be quantified by $2 \mathrm{D}$ and 3D ultrasound imaging in several xenograft models. Pezold et al. [41] demonstrated in a murine orthotopic oral cavity tumor model that tumor dimensions acquired with ultrasound measurements were not significantly different compared to histological measurement [42]. Moreover, highfrequency ultrasound allows noninvasive longitudinal assays [17]; UBM detects small tumor nodules in early stage with lower limit of detection at approximately $0.4 \mathrm{~mm}$ in volume (Figure 8). In a model of thyroid cancer UBM detected the presence of malignant thyroid nodules quite early, long before they were palpable, and UBM results were compared with histological findings [2].

In a model of human mammary cancer, 3D was used to monitor tumor growth every week and to detect tumor response after therapy, showing a better correlation with postmortem findings in comparison to other imaging technique like bioluminescence and positron emission tomography [43] (Figure 9).
Recently, Banihashemi et al. [44] demonstrated that UBM is able to detect apoptotic cell death in a preclinical tumor model of melanoma after treatment with photodynamic therapy in models of bladder and colorectal cancer.

Power Doppler ultrasound captures flow-dependent signals in blood vessels and can be used to assess neoangiogenesis. However, its sensitivity is limited to vessels larger than capillaries. Xuan et al. [13] reported the first application of high-frequency three-dimensional power Doppler ultrasound imaging in a genetically engineered mouse prostate cancer model. Tumor vascularity was quantified in power Doppler images by computing the color pixel density for the entire tumor and the peripheral part of the prostate tumor. The vascular architecture at different stages of tumor growth was detected comparing 3D power Doppler with contrast-enhanced micro-CT. Comparisons suggested that the smallest vessels reliably detected by power Doppler were from 100 to $150 \mu \mathrm{m}$ diameter. Contrast agents revealed all perfused vessels by assessing stimulated acoustic emissions from microbubbles. Angiogenesis can be enhanced in neoplastic tissues by several mechanisms such as overexpression of angiogenetic factors or mobilization of angiogenetic proteins in the extracellular matrix [45], and the amount of new blood vessel growth is correlated with poor prognosis in several tumor types [46]. Therefore, there is a great interest in the development of antiangiogenetic drugs such as antiVEGF treatments and of imaging techniques that could be helpful in quantifying tumor vasculature and the VEGF expression.

More recently, Willman et al. [35] demonstrated that angiogenesis is detected in angiosarcoma and malignant glioma in mice using microbubbles labeled with monoclonal antibodies against murine VEGF-R2. A dual-targeted contrast-enhanced US directed at both VEGF-R2 and alpha(v)beta(3) integrin improves in vivo visualization of tumor angiogenesis in a human ovarian cancer xenograft tumor model in mice.

A major challenge for tumor therapy is to obtain effects directly into an affected tissue of nucleic acids delivered in systemic circulation. Intravenous injections of a replication incompetent adenovirus with a cytokine displaying cancer selective-apoptosis profoundly inhibits prostate cancer growth in animals in which the tumor was insonated in comparison with the control groups injected with adenovirus but not insonated. These findings demonstrate the utility and the potential therapeutic applications of novel microbubbles guided gene therapy technology [7].

7.2. Cardiology. UBM provide an opportunity to characterize anatomy and physiology of heart and great vessels in a safe, reliable, and noninvasive modality in adult mice.

$2 \mathrm{D}$ and 3D imaging have been used to evaluate cardiac structure and valves morphology and function in mice $[1,47,48]$, to calculate cardiac function parameters such as stroke volume, cardiac output, ejection fraction, and fractional shortening, and to assess global left ventricular function and LV mass $[8,47-49]$ in normal and in models 


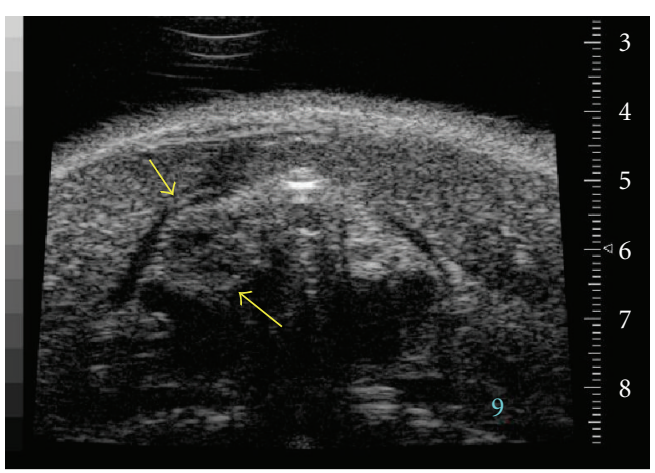

(a)

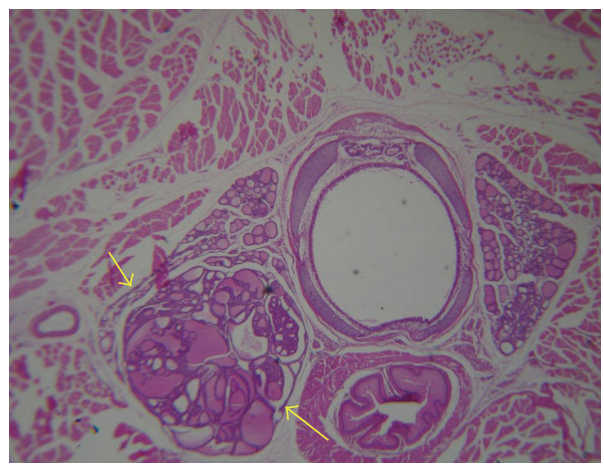

(b)

FIGURE 8: (a) UBM microimaging of thyroid in living mice performed with a $35 \mathrm{MHz}$ probe in a genetically engineered mice model of thyroid disease (TRK). The figure shows an enlarged left lobe with hypoechoic nodule (arrows). (b) Histopathology analysis shows a thyroid adenoma. From Mancini et al. Endocrinology [2].

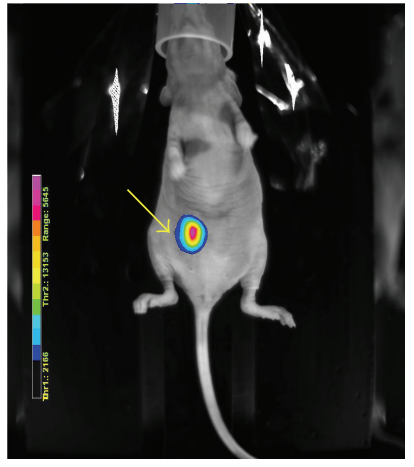

(a) Bioluminescence

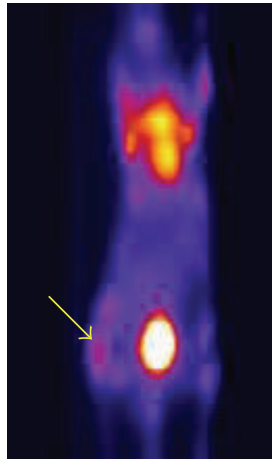

(b) PET

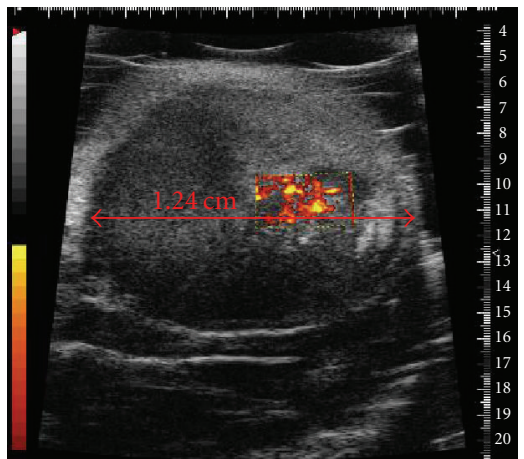

(c) UBM

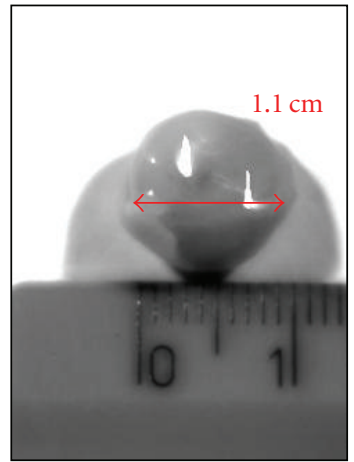

(d) Necroscopy

FIGURE 9: Multimodality imaging of the lesion: human mammary tumor cells were implanted in the mammary fat pad of an athymic nude mice, 6 weeks old. There is a good correspondence between the morphology and the size of the tumor detected by UBM and necroscopic findings. (a) Bioluminescence of the tumor. (b) ${ }^{18} \mathrm{~F}$-flourothymidine total body PET, tumor uptake is evident (arrow). (c) UBM with measurements of neoplasia. (d) Post mortem dimension of the explanted tumor.

of cardiovascular diseases [50-54]. Pharmacological effects of various agents tested in mouse models have also been evaluated with echocardiography $[55,56]$.

Small heart size hampers accurate injection into the left ventricular (LV) wall and require surgical visualization of the heart, but that, however, do not prevent unintentional injection into the LV cavity. High-resolution echocardiography is able to guide cardiac injections accurately into the myocardial wall of mice with closet chest. This system was successful to target the injection of labeled cells into specific cardiac regions such as myocardium adjacent to ischemic area in a mouse myocardial infarction model or to treat ischemic myocardium implanting bone marrow cells and to guide left ventricular catheterization $[9,10]$.

Microbubbles have been applied mainly as blood pool agents to enhance the echogenicity of the cardiac chambers during echocardiography for better delineation of the myocardial borders and for perfusion assessment of the myocardium [57].

Using 2D B-mode imaging, fetal heart development can be detected in mice as early as embryonic day 8.5 , when the linear heart tube begins to beat. On day 9.5, the U-shaped heart tube is clearly visible, and Doppler blood velocity waveforms can be recorded separately from the inflow and outflow regions of the heart tube (Figure 10(a)). On day 11.5, it is possible to detect the process of division of the outflow tract into the ascending aorta and main pulmonary artery (Figure 10(b)). At E12.5, the separation of the aorta and main pulmonary artery appears complete, but the interventricular septum is visibly incomplete, and flow streams from both ventricles can be seen entering the aorta (Figure 10(c)). At day 13.5 , the embryonic ventricles are fully separated by a septum, the atrioventricular valves are visible, and the heart has a mature fetal form (Figure 10(d)). After day 15.5, the heart chambers begin to darken in the ultrasound image, and the ventricular wall, endocardium, and septum are easier to be discerned (Figure 10(e)). The improved contrast seen after day 15.5 means that ventricular chamber dimensions and wall thickness measurements become feasible $[5,58]$. Fetal blood is echodense at UBM frequencies, due to nucleated erythroblasts, and this may facilitate assessment of major vascular development. 


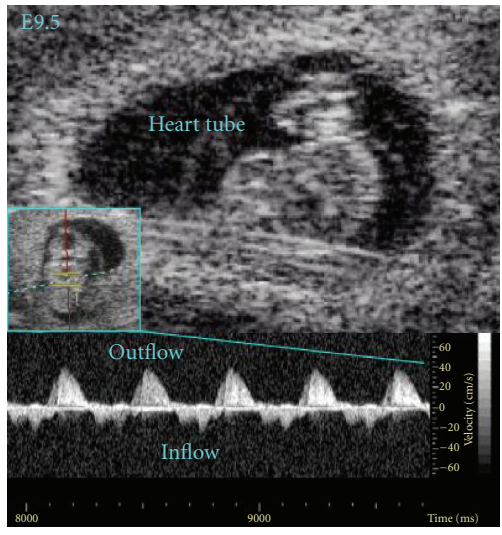

(a)

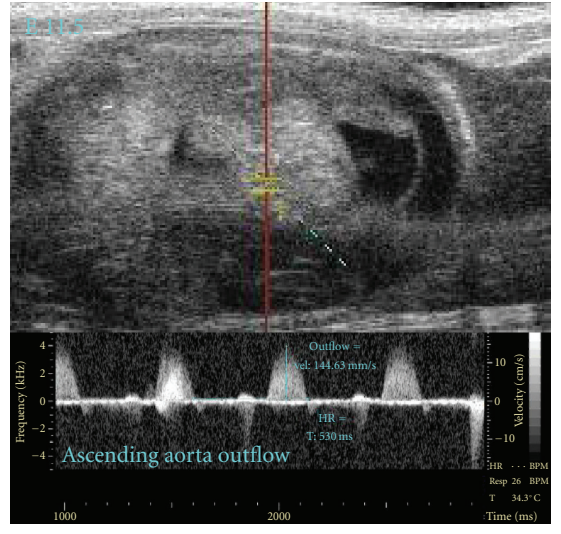

(b)

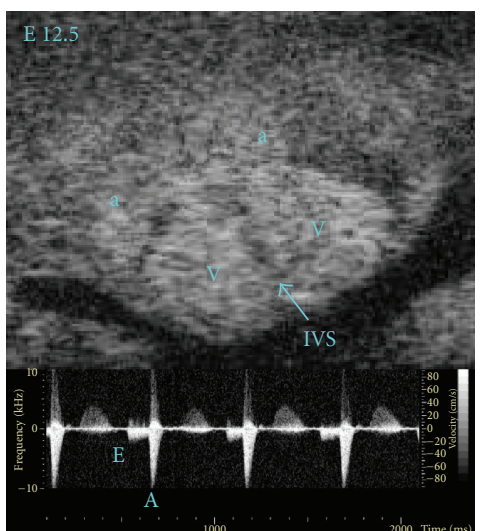

(c)

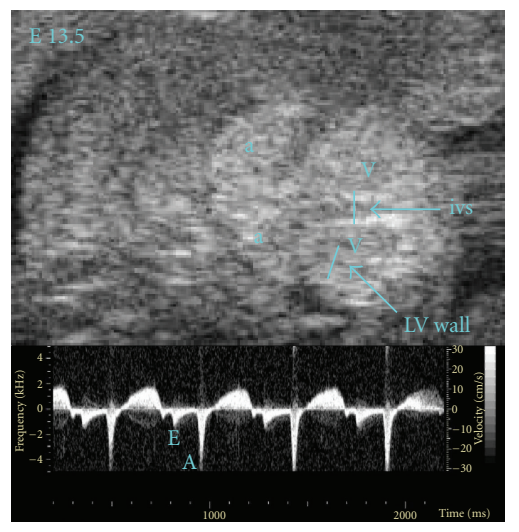

(d)

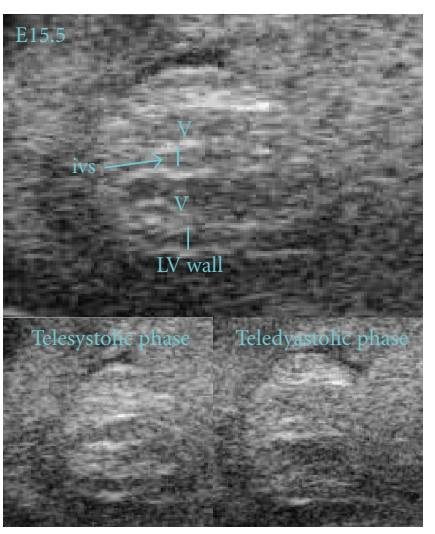

(e)

Figure 10: (a) E9.5: heart tube and doppler spectral trace of ventricular inflow and outflow at 9.5 gestational age. (b) E11.5: doppler spectral trace of ascending aorta outflow at 11.5 gestational age. (c) E12.5: bidimensional image of atrio-ventricular chambers (a-V) interventricular septum (IVS); and doppler spectral trace of ascending aorta outflow at 12.5 gestational age. (d) E13.5: bidimensional image of atrioventricular chambers, interventricular septum, and left ventricle wall (LV wall); doppler spectral trace of ventricular inflow and outflow at 13.5 gestational age. The atrial wave (A-wave) was dominant. The rapid ventricular filling (E-wave) is a measure of ventricular compliance. (e) E15.5: bidimensional image of ventricular chambers, interventricular septum, and left ventricle wall in telesystolic and telediastolic fase of heart cycle in a 15.5 days mouse embryo.

Noninvasive transthoracic echocardiography in newborn C57BL mice has been shown to define normal cardiac and great vessel anatomic measurements [59].

The mouse has become a powerful tool for studying genetic models of cardiac development and congenital heart disease and echocardiography is an ideal method for detecting and studying congenital malformations in living fetus, as it allows early recognition of abnormalities and the progression of disease can be followed in utero with longitudinal studies $[5,48,59,60]$.

7.3. Developmental Biology. In utero imaging of live mouse embryos allows studies of early embryonic developmental stages, embryonic neural tubes and heart development, and the effects of mutant phenotypes during embryogenesis.

UBM resolution is sufficient to visualize small anatomical structures, especially at embryonic and early postnatal stages [61] and to study the morphology of various organ systems throughout development.

The feasibility of UBM for in vivo morphometric quantification of embryonic growth and for estimating gestational age and embryonic body weight in utero as discussed elsewhere $[62,63]$.

At the earlier stage (E11.5), major features of the embryo can be identified, including amniotic membrane and cavity, yolk sac, placenta, umbilical vessels, and heart and brain ventricles. At later stages (>E13.5), brain, mouth, eyes, lungs, heart chambers, liver, kidneys, chest wall, spine, limbs, and tail are also clearly visualized.

In the semi-invasive approach, a small incision is made in the abdominal wall, and one or two embryos are exteriorized. It has the advantage to exclude the maternal abdominal wall, and intra-abdominal content to provide a better resolution of embryonic structures.

Doppler flow in the developing brain has also been obtained $[59,64]$. Investigations about eyes limbs and spine and skin oculodentodigital dysplasias in mouse embryos have also been reported using this technology [39, 40, 65].

7.4. Ocular Applications. UBM is a good tool for imaging the anterior ocular segment anatomy and pathology, including the cornea, anterior chamber, iris, ciliary body, and lens 
[31, 32, 34, 36, 66-69]. Throughout development until E18.5, anterior segment structures appear compressed, revealing little structural detail or differentiation from surrounding tissues. It is possible to perform several quantitative analysis: measurements of the corneal thickness, of the anterior chamber depth, of the trabecular-iris angle, and of the iridozonular distance. It is, thus, applicable for diagnostic imaging of corneal diseases, glaucoma, cysts, and tumors. Imaging of posterior structures of the eye, and in particular, the retina and the optical nerve can also be obtained. Normal embryonic development of the mouse eye can be studied by ultrasound biomicroscopy with a focus on the formation of the retina, lens, and cornea. The earliest stages of development that can be successfully imaged are at approximately E8.5, coinciding with the appearance of the optic placode and vesicle. At this point, the morphogenesis of the eye is very primitive, and features of the developing tissues are close to the resolution limit of the scanner. Subsequent development of the lens vesicle, retina, cornea, vitreous, and conjunctiva can be observed up to the birth of the mouse. No evidence of flow has yet been observed in the developing prenatal mouse eye.

\section{Conclusions}

Advantages of ultrasound imaging over other imaging techniques that use ionizing radiation (e.g., PET, SPECT, and CT) is that it less invasive, does not require specialized ancillary equipments, is safer for the animals and the operators, and, finally, is a low-cost technology.

However, ultrasound imaging has also some limitations as a research tool. In fact, it requires the knowledge and expertise of a well-trained sonographer to obtain accurate and repeatable images and is limited by bone- and gas-filled structures; therefore, ultrasound is not routinely used in brain, spinal cord, and lung imaging. The development of novel targeted contrast agents will foster future applications to study the molecular basis of animal models. UBM provide a noninvasive imaging of both embryos and adult mice anatomy and pathology. It is an excellent tool for cancer research and cardiovascular investigations to analyze ocular and abdominal structures and embryos development.

\section{Acknowledgment}

A. Brunetti and M. Salvatore contribute equally to this work.

\section{References}

[1] R. B. Hinton, C. M. Alfieri, S. A. Witt et al., "Mouse heart valve structure and function: echocardiographic and morphometric analyses from the fetus through the aged adult," American Journal of Physiology-Heart and Circulatory Physiology, vol. 294, no. 6, pp. H2480-H2488, 2008.

[2] M. Mancini, E. Vergara, G. Salvatore et al., "Morphological ultrasound microimaging of thyroid in living mice," Endocrinology, vol. 150, no. 10, pp. 4810-4815, 2009.

[3] Z. P. Shen, A. A. Brayman, L. Chen, and C. H. Miao, "Ultrasound with microbubbles enhances gene expression of plasmid DNA in the liver via intraportal delivery," Gene Therapy, vol. 15, no. 16, pp. 1147-1155, 2008.

[4] F. S. Foster, M. Zhang, A. S. Duckett, V. Cucevic, and C. J. Pavlin, "In vivo imaging of embryonic development in the mouse eye by ultrasound biomicroscopy," Investigative Ophthalmology and Visual Science, vol. 44, no. 6, pp. 23612366, 2003.

[5] C. F. Spurney, C. W. Lo, and L. Leatherbury, "Fetal mouse imaging using echocardiography: a review of current technology," Echocardiography, vol. 23, no. 10, pp. 891-899, 2006.

[6] D. H. Turnbull, T. S. Bloomfield, H. S. Baldwin, F. S. Foster, and A. L. Joyner, "Ultrasound backscatter microscope analysis of early mouse embryonic brain development," Proceedings of the National Academy of Sciences of the United States of America, vol. 92, no. 6, pp. 2239-2243, 1995.

[7] A. Greco, A. Di Benedetto, C. M. Howard et al., "Eradication of therapy-resistant human prostate tumors using an ultrasound-guided site-specific cancer terminator virus delivery approach," Molecular Therapy, vol. 18, no. 2, pp. 295$306,2010$.

[8] H. C. Manning, N. B. Merchant, A. C. Foutch et al., "Molecular imaging of therapeutic response to epidermal growth factor receptor blockade in colorectal cancer," Clinical Cancer Research, vol. 14, no. 22, pp. 7413-7422, 2008.

[9] M. L. Springer, R. E. Sievers, M. N. Viswanathan et al., "Closed-chest cell injections into mouse myocardium guided by high-resolution echocardiography," American Journal of Physiology-Heart and Circulatory Physiology, vol. 289, no. 3, pp. H1307-H1314, 2005.

[10] Y. Q. Zhou, L. Davidson, R. M. Henkelman et al., "Ultrasound-guided left-ventricular catheterization: a novel method of whole mouse perfusion for microimaging," Laboratory Investigation, vol. 84, no. 3, pp. 385-389, 2004.

[11] A. Greco, S. E. Kelly, P. P. Claudio, M. Salvatore, and A. Brunetti, "Ultrasound Contrast Agents for imaging and gene therapy," in Proceedings of the AISAL Symposium "Imaging and Translational Medicine, p. 25, 2010.

[12] F. S. Foster, M. Y. Zhang, Y. Q. Zhou et al., "A new ultrasound instrument for in vivo microimaging of mice," Ultrasound in Medicine and Biology, vol. 28, no. 9, pp. 1165-1172, 2002.

[13] J. W. Xuan, M. Bygrave, H. Jiang et al., "Functional neoangiogenesis imaging of genetically engineered mouse prostate cancer using three-dimensional power Doppler ultrasound," Cancer Research, vol. 67, no. 6, pp. 2830-2839, 2007.

[14] O. Balogun, G. D. Cole, R. Huber, D. Chinn, T. W. Murray, and J. B. Spicer, "High-spatial-resolution sub-surface imaging using a laser-based acoustic microscopy technique," IEEE Transactions on Ultrasonics, Ferroelectrics, and Frequency Control, vol. 58, no. 1, pp. 226-233, 2011.

[15] B. E. Treeby, E. Z. Zhang, A. S. Thomas, and B. T. Cox, "Measurement of the ultrasound attenuation and dispersion in whole human blood and its components from 0-70 MHz," Ultrasound in Medicine and Biology, vol. 37, no. 2, pp. 289300, 2011.

[16] M. Tamaki, K. Kidoguchi, T. Mizobe et al., "Carotid artery occlusion and collateral circulation in C57Black/6J mice detected by synchrotron radiation microangiography," Kobe Journal of Medical Sciences, vol. 52, no. 5, pp. 111-118, 2006.

[17] K. C. Graham, L. A. Wirtzfeld, L. T. MacKenzie et al., "Threedimensional high-frequency ultrasound imaging for longitudinal evaluation of liver metastases in preclinical models," Cancer Research, vol. 65, no. 12, pp. 5231-5237, 2005.

[18] L. A. Wirtzfeld, K. C. Graham, A. C. Groom et al., "Volume measurement variability in three-dimensional high-frequency 
ultrasound images of murine liver metastases," Physics in Medicine and Biology, vol. 51, no. 10, pp. 2367-2381, 2006.

[19] J. R. Lindner, "Microbubbles in medical imaging: current applications and future directions," Nature Reviews Drug Discovery, vol. 3, no. 6, pp. 527-532, 2004.

[20] F. S. Villanueva, R. J. Jankowski, S. Klibanov et al., "Microbubbles targeted to intercellular adhesion molecule-1 bind to activated coronary artery endothelial cells," Circulation, vol. 98, no. 1, pp. 1-5, 1998.

[21] B. Walzog, D. Schuppan, C. Heimpel, A. Hafezi-Moghadam, P. Gaehtgens, and K. Ley, "The leukocyte integrin Mac-1 (CD11b/CD18) contributes to binding of human granulocytes to collagen," Experimental Cell Research, vol. 218, no. 1, pp. 28 38, 1995.

[22] M. Mancini, A. Greco, A. Speranza, and M. Salvatore, "Ultrasound molecular imaging by targeted microbubble contrast agents," Minerva Biotecnologica, vol. 21, no. 2, pp. 97-110, 2009.

[23] H. D. Liang and M. J. K. Blomley, "The role of ultrasound in molecular imaging," British Journal of Radiology, vol. 76, no. 2, pp. S140-S150, 2003.

[24] S. L. Huang, "Liposomes in ultrasonic drug and gene delivery," Advanced Drug Delivery Reviews, vol. 60, no. 10, pp. 11671176, 2008.

[25] C. K. Holland and D. D. McPherson, "Echogenic lipsomes for targeted drug delivery," in Proceedings IEEE International Symposium of Biomedical Imaging (ISBI '09), pp. 755-758, July 2009.

[26] G. M. Lanza and S. A. Wickline, "Targeted ultrasonic contrast agents for molecular imaging and therapy," Progress in Cardiovascular Diseases, vol. 44, no. 1, pp. 13-31, 2001.

[27] O. Couture, P. D. Bevan, E. Cherin, K. Cheung, P. N. Burns, and F. S. Foster, "Investigating perfluorohexane particles with high-frequency ultrasound," Ultrasound in Medicine and Biology, vol. 32, no. 1, pp. 73-82, 2006.

[28] N. Reznik, R. Williams, and P. N. Burns, "Investigation of vaporized submicron perfluorocarbon droplets as an ultrasound contrast agent," Ultrasound in Medicine and Biology, vol. 37, no. 8, pp. 1271-1279, 2011.

[29] R. Bekeredjian, S. Behrens, J. Ruef et al., "Potential of goldbound microtubules as a new ultrasound contrast agent," Ultrasound in Medicine and Biology, vol. 28, no. 5, pp. 691695, 2002.

[30] H. A. Bardelmeijer, T. Buckle, M. Ouwehand, J. H. Beijnen, J. H. M. Schellens, and O. van Tellingen, "Cannulation of the jugular vein in mice: a method for serial withdrawal of blood samples," Laboratory Animals, vol. 37, no. 3, pp. 181187, 2003.

[31] P. Haag, F. Frauscher, J. Gradl et al., "Microbubble-enhanced ultrasound to deliver an antisense oligodeoxynucleotide targeting the human androgen receptor into prostate tumours," Journal of Steroid Biochemistry and Molecular Biology, vol. 102, no. 1-5, pp. 103-113, 2006.

[32] Y. Miyake, K. Ohmori, J. Yoshida et al., "Granulocyte colonystimulating factor facilitates the angiogenesis induced by ultrasonic microbubble destruction," Ultrasound in Medicine and Biology, vol. 33, no. 11, pp. 1796-1804, 2007.

[33] J. C. Slevin, L. Byers, M. Gertsenstein et al., "High resolution ultrasound-guided microinjection for interventional studies of early embryonic and placental development in vivo in mice," BMC Developmental Biology, vol. 27, no. 6, p. 10, 2006.

[34] D. Guo, X. Li, P. Sun et al., "Ultrasound/microbubble enhances foreign gene expression in ECV304 cells and murine myocardium," Acta Biochimica et Biophysica Sinica, vol. 36, no. 12, pp. 824-831, 2004.

[35] J. K. Willmann, A. M. Lutz, R. Paulmurugan et al., "Dualtargeted contrast agent for US assessment of tumor angiogenesis in vivo," Radiology, vol. 248, no. 3, pp. 936-944, 2008.

[36] A. Lyshchik, A. C. Fleischer, J. Huamani, D. E. Hallahan, M. Brissova, and J. C. Gore, "Molecular imaging of vascular endothelial growth factor receptor 2 expression using targeted contrast-enhanced high-frequency ultrasonography," Journal of Ultrasound in Medicine, vol. 26, no. 11, pp. 1575-1586, 2007.

[37] C. M. Howard, F. Forsberg, C. Minimo, J. B. Liu, D. A. Merton, and P. P. Claudio, "Ultrasound guided site specific gene delivery system using adenoviral vectors and commercial ultrasound contrast agents," Journal of Cellular Physiology, vol. 209, no. 2, pp. 413-421, 2006.

[38] M. R. Böhmer, A. L. Klibanov, K. Tiemann, C. S. Hall, H. Gruell, and O. C. Steinbach, "Ultrasound triggered imageguided drug delivery," European Journal of Radiology, vol. 70, no. 2, pp. 242-253, 2009.

[39] R. Pla, V. Borrell, N. Flames, and O. Marín, "Layer acquisition by cortical GABAergic interneurons is independent of Reelin signaling," Journal of Neuroscience, vol. 28, no. 26, pp. 69246934, 2006.

[40] C. Punzo and C. L. Cepko, "Ultrasound-guided in utero injections allow studies of the development and function of the eye," Developmental Dynamics, vol. 237, no. 4, pp. 10341042, 2008.

[41] J. C. Pezold, K. Zinn, M. A. Talbert, R. Desmond, and E. L. Rosenthal, "Validation of ultrasonography to evaluate murine orthotopic oral cavity tumors," ORL, vol. 68, no. 3, pp. 159163, 2006.

[42] C. P. Chang, L. Chen, and G. R. Crabtree, "Sonographic staging of the developmental status of mouse embryos in utero," Genesis, vol. 36, no. 1, pp. 7-11, 2003.

[43] M. Zollo, N. Marino, A. Greco et al., "In vivo imaging methodologies applied to the murine xenografts of human breast cancer: a pre-clinical h-prune animal model," in Proceedings of the European Symposium of Molecular Imaging, p. 38, Naples, Italy, May 2007.

[44] B. Banihashemi, R. Vlad, B. Debeljevic, A. Giles, M. C. Kolios, and G. J. Czarnota, "Ultrasound imaging of apoptosis in tumor response: novel preclinical monitoring of photodynamic therapy effects," Cancer Research, vol. 68, no. 20, pp. 8590-8596, 2008.

[45] J. Folkman, "What is the evidence that tumors are angiogenesis dependent?" Journal of the National Cancer Institute, vol. 82, no. 1, pp. 4-6, 1990.

[46] C. Dazzi, A. Cariello, P. Maioli et al., "Prognostic and predictive value of intratumoral microvessels density in operable non-small-cell lung cancer," Lung Cancer, vol. 24, no. 2, pp. 81-88, 1999.

[47] M. Scherrer-Crosbie and H. B. Thibault, "Echocardiography in translational research: of mice and men," Journal of the American Society of Echocardiography, vol. 21, no. 10, pp. 1083-1092, 2008.

[48] J. Stypmann, M. A. Engelen, C. Troatz, M. Rothenburger, L. Eckardt, and K. Tiemann, "Echocardiographic assessment of global left ventricular function in mice," Laboratory Animals, vol. 43, no. 2, pp. 127-137, 2009.

[49] R. C. Fentzke, C. E. Korcarz, S. G. Shroff et al., "Evaluation of ventricular and arterial hemodynamics in anesthetized closedchest mice," Journal of the American Society of Echocardiography, vol. 10, no. 9, pp. 915-925, 1997. 
[50] P. Krishnamurthy, J. Rajasingh, E. Lambers, G. Qin, D. W. Losordo, and R. Kishore, "IL-10 inhibits inflammation and attenuates left ventricular remodeling after myocardial infarction via activation of STAT3 and suppression of HuR," Circulation Research, vol. 104, no. 2, pp. e9-e18, 2009.

[51] J. Takagawa, Y. Zhang, M. L. Wong et al., "Myocardial infarct size measurement in the mouse chronic infarction model: comparison of area- and length-based approaches," Journal of Applied Physiology, vol. 102, no. 6, pp. 2104-2111, 2007.

[52] T. P. Abraham, M. Jones, K. Kazmierczak et al., "Diastolic dysfunction in familial hypertrophic cardiomyopathy transgenic model mice," Cardiovascular Research, vol. 82, no. 1, pp. 8492, 2009.

[53] L. Gavish, C. Rubinstein, A. Bulut et al., "Low-level laser irradiation inhibits abdominal aortic aneurysm progression in apolipoprotein E-deficient mice," Cardiovascular Research, vol. 83, no. 4, pp. 785-792, 2009.

[54] M. Ni, M. Zhang, S. F. Ding, W. Q. Chen, and Y. Zhang, "Micro-ultrasound imaging assessment of carotid plaque characteristics in apolipoprotein-E knockout mice," Atherosclerosis, vol. 197, no. 1, pp. 64-71, 2008.

[55] M. Walker, B. R. Campbell, K. Azer et al., "A novel 3dimensional micro-ultrasound approach to automated measurement of carotid arterial plaque volume as a biomarker for experimental atherosclerosis," Atherosclerosis, vol. 204, no. 1, pp. 55-65, 2009.

[56] G. Bragagni, F. Lari, G. Magenta, R. Brogna, and G. Zoli, "Echocardiographic evaluation of anti-tumor necrosis factor$\alpha$ therapy with infliximab in patients without cardiac pathologies," Recenti Progressi in Medicina, vol. 101, no. 7-8, pp. 289292, 2010.

[57] J. Grönros, J. Wikström, U. Brandt-Eliasson et al., "Effects of rosuvastatin on cardiovascular morphology and function in an ApoE-knockout mouse model of atherosclerosis," American Journal of Physiology - Heart and Circulatory Physiology, vol. 295, no. 5, pp. H2046-H2053, 2008.

[58] B. A. Kaufmann, M. Lankford, C. Z. Behm et al., "Highresolution myocardial perfusion imaging in mice with highfrequency echocardiographic detection of a depot contrast agent," Journal of the American Society of Echocardiography, vol. 20, no. 2, pp. 136-143, 2007.

[59] S. Srinivasan, H. S. Baldwin, O. Aristizabal et al., "Noninvasive, in utero imaging of mouse embryonic heart development with 40-MHz echocardiography," Circulation, vol. 98, no. 9, pp. 912-918, 1998.

[60] C. K. L. Phoon, R. P. Ji, O. Aristizábal et al., "Embryonic heart failure in NFATc1-/- mice novel: mechanistic insights from in utero ultrasound biomicroscopy," Circulation Research, vol. 95, no. 1, pp. 92-99, 2004.

[61] N. Corrigan, D. P. Brazil, and F. M. Auliffe, "High-frequency ultrasound assessment of the murine heart from embryo through to juvenile," Reproductive Sciences, vol. 17, no. 2, pp. 147-157, 2010.

[62] D. H. Turnbull, J. A. Ramsay, G. S. Shivji et al., "Ultrasound backscatter microscope analysis of mouse melanoma progression," Ultrasound in Medicine and Biology, vol. 22, no. 7, pp. 845-853, 1996.

[63] M. Russo, L. Meomartino, A. Greco et al., "Pregnancy detection in mice using ultrasound," Veterinary Record, vol. 160, no. 13, pp. 446-447, 2007.

[64] J. Mu, J. C. Slevin, D. Qu, S. McCormick, and S. L. Adamson, "In vivo quantification of embryonic and placental growth during gestation in mice using micro-ultrasound,"
Reproductive Biology and Endocrinology, vol. 12, no. 6, p. 34, 2008.

[65] D. H. Turnbull, "In utero ultrasound backscatter microscopy of early stage mouse embryos," Computerized Medical Imaging and Graphics, vol. 23, no. 1, pp. 25-31, 1999.

[66] E. Bentley, P. E. Miller, and K. A. Diehl, "Use of highresolution ultrasound as a diagnostic tool in veterinary ophthalmology," Journal of the American Veterinary Medical Association, vol. 223, no. 11, pp. 1617-1622, 2003.

[67] A. M. Flenniken, L. R. Osborne, N. Anderson et al., "A Gja1 missense mutation in a mouse model of oculodentodigital dysplasia," Development, vol. 132, no. 19, pp. 4375-4386, 2005.

[68] N. Nissirios, J. Ramos-Esteban, and J. Danias, "Ultrasound biomicroscopy of the rat eye: effects of cholinergic and anticholinergic agents," Graefe's Archive for Clinical and Experimental Ophthalmology, vol. 243, no. 5, pp. 469-473, 2005.

[69] S. W. M. John, R. S. Smith, O. V. Savinova et al., "Essential iris atrophy, pigment dispersion, and glaucoma in DBA/2J mice," Investigative Ophthalmology and Visual Science, vol. 39, no. 6, pp. 951-962, 1998. 


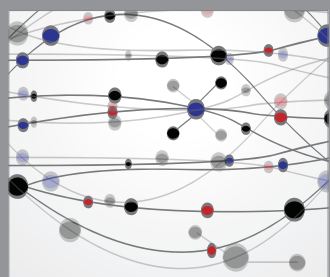

The Scientific World Journal
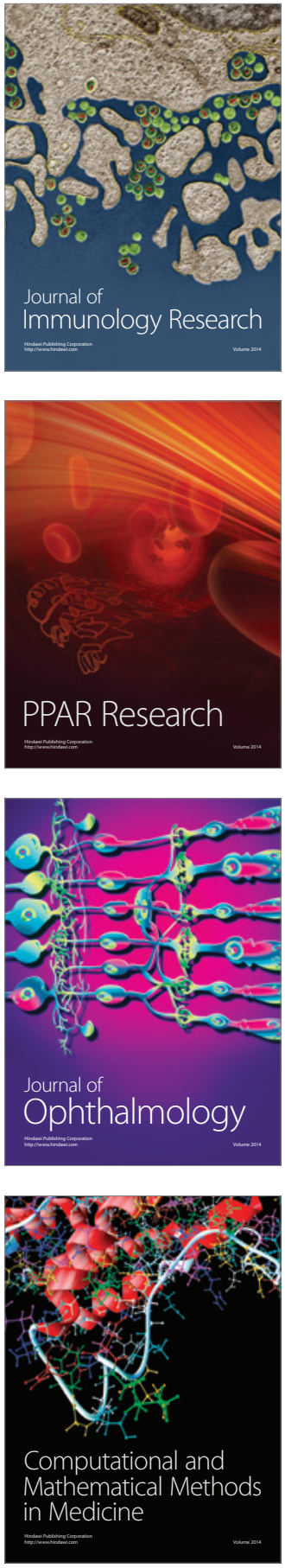

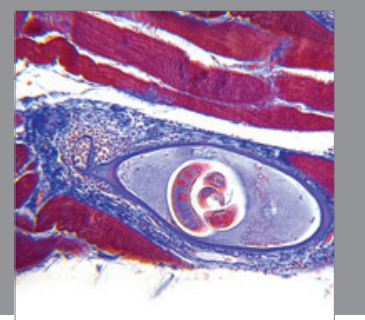

Gastroenterology

Research and Practice
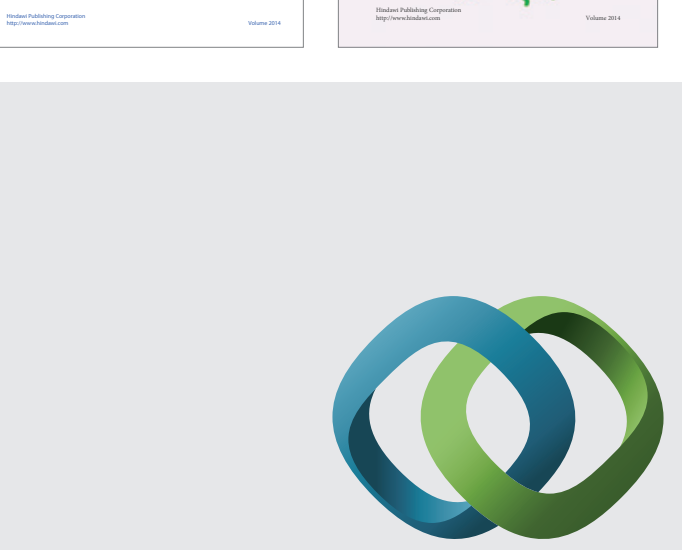

\section{Hindawi}

Submit your manuscripts at

http://www.hindawi.com
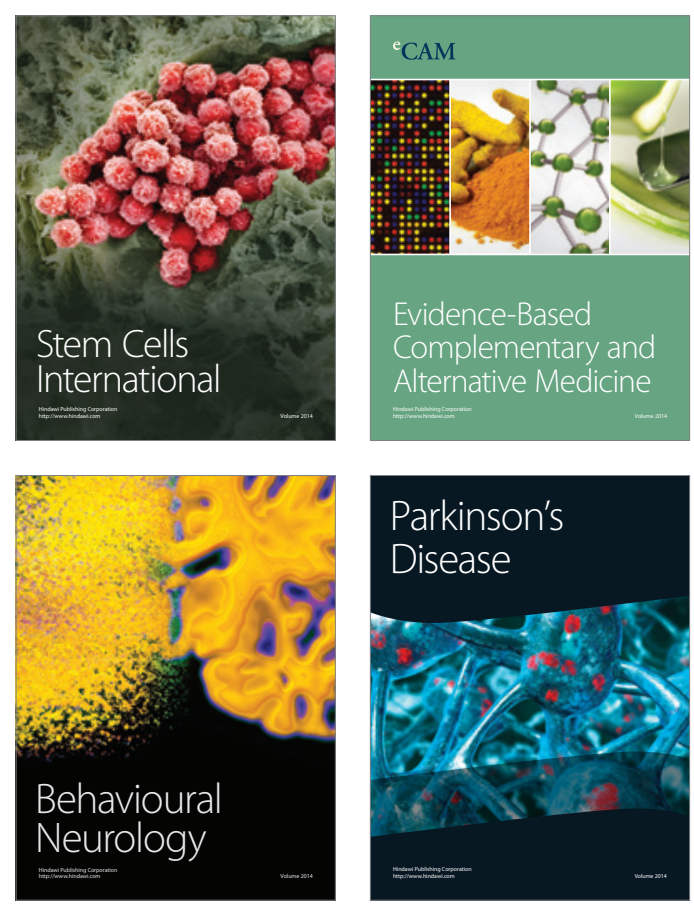

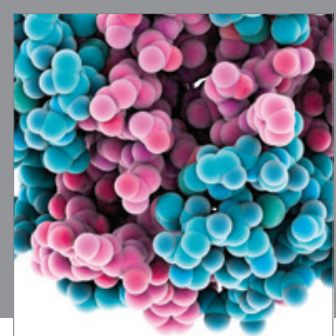

Journal of
Diabetes Research

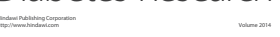

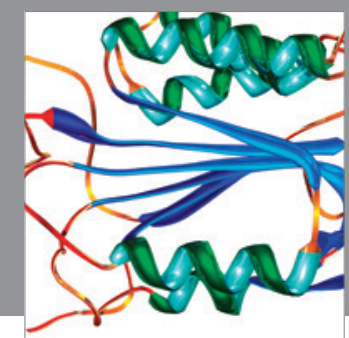

Disease Markers
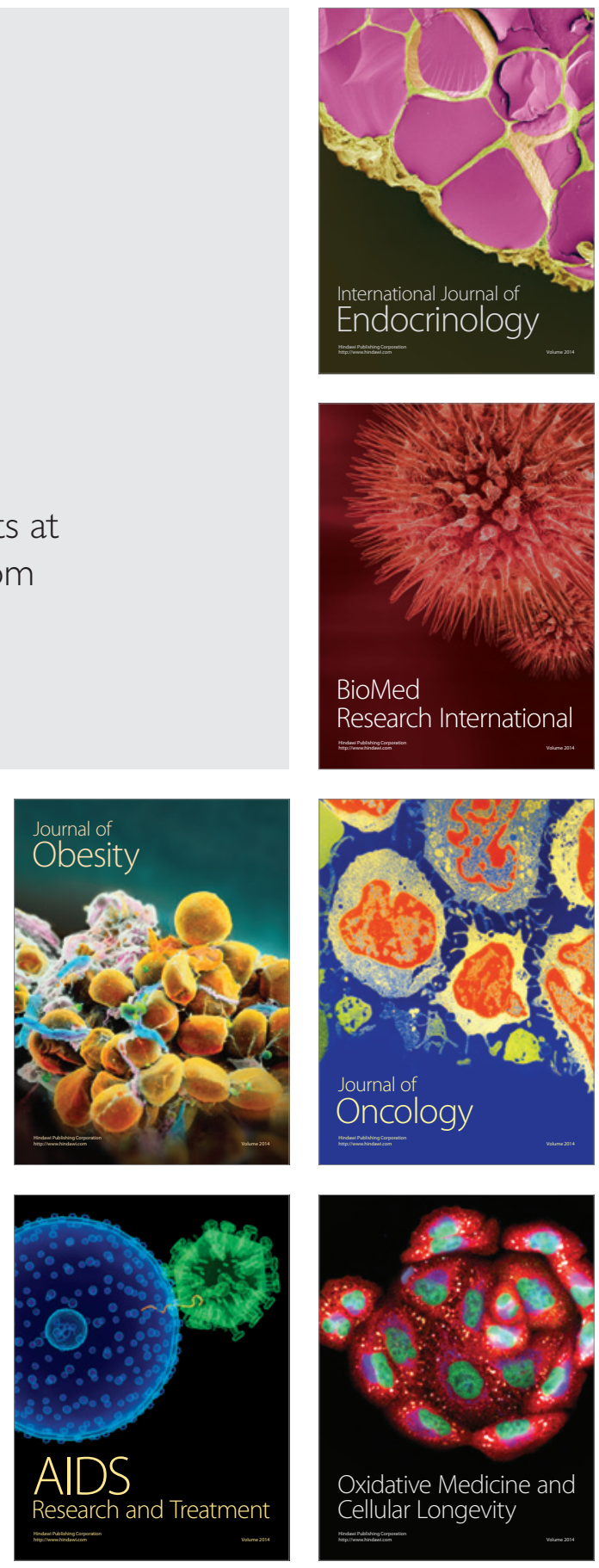